\title{
Targeting experimental orthotopic glioblastoma with chitosan-based superparamagnetic iron oxide nanoparticles (CS-DX-SPIONs)
}

This article was published in the following Dove Press journal: International Journal of Nanomedicine

\author{
Maxim Shevtsov ${ }^{1-4}$ \\ Boris Nikolaev ${ }^{5}$ \\ Yaroslav Marchenko ${ }^{5}$ \\ Ludmila Yakovleva $^{5}$ \\ Nikita Skvortsov ${ }^{5}$ \\ Anton Mazur ${ }^{6}$ \\ Peter Tolstoy ${ }^{6}$ \\ Vyacheslav Ryzhov ${ }^{7}$ \\ Gabriele Multhoff ${ }^{2}$ \\ 'Department of Cell Biotechnology, \\ Institute of Cytology of the Russian \\ Academy of Sciences, St Petersburg, \\ Russia; ${ }^{2}$ Department of Radiation \\ Immuno Oncology, Technische \\ Universität München, Klinikum \\ rechts der Isar, Munich, Germany; \\ ${ }^{3}$ Department of Biotechnology, \\ Pavlov First Saint Petersburg State \\ Medical University, St Petersburg, \\ Russia; ${ }^{4}$ Department of Pediatric \\ Neurosurgery, Polenov Russian \\ Scientific Research Institute of \\ Neurosurgery, St Petersburg, Russia; \\ ${ }^{5}$ Department of Nanomedicine, \\ Research Institute of Highly Pure \\ Biopreparations, St Petersburg, \\ Russia; ${ }^{6}$ Department of NMR, Saint \\ Petersburg State University, St \\ Petersburg, Russia; ' Department of \\ NMR, NRC "Kurchatov Institute", \\ Petersburg Nuclear Physics Institute, \\ Gatchina, Russia
}

Correspondence: Maxim Shevtsov Technische Universität München, Klinikum rechts der Isar, 8I675 Ismaniger Street 22, Munich, Germany

Tel +49 I7 $4442 \quad 1888$

Fax +4989 4I 404299

Email maxim.shevtsov@tum.de
Background: Glioblastoma is the most devastating primary brain tumor of the central nervous system in adults. Magnetic nanocarriers may help not only for a targeted delivery of chemotherapeutic agents into the tumor site but also provide contrast enhancing properties for diagnostics using magnetic resonance imaging (MRI).

Methods: Synthesized hybrid chitosan-dextran superparamagnetic nanoparticles (CS-DXSPIONs) were characterized using transmission electron microscopy (TEM) and relaxometry studies. Nonlinear magnetic response measurements were employed for confirming the superparamagnetic state of particles. Following in vitro analysis of nanoparticles cellular uptake tumor targeting was assessed in the model of the orthotopic glioma in rodents.

Results: CS-DX-SPIONs nanoparticles showed a uniform diameter of $55 \mathrm{~nm}$ under TEM and superparamagentic characteristics as determined by $T_{1}$ (spin-lattice relaxation time) and $T_{2}$ (spinspin relaxation time) proton relaxation times. Application of the chitosan increased the charge from +8.9 to $+19.3 \mathrm{mV}$ of the dextran-based SPIONs. The nonlinear magnetic response at second harmonic of CS-DX-SPIONs following the slow change of stationary magnetic fields with very low hysteresis evidenced superparamagnetic state of particles at ambient temperatures. Confocal microscopy and flow cytometry studies showed an enhanced internalization of the chitosanbased nanoparticles in U87, C6 glioma and HeLa cells as compared to dextran-coated particles. Cytotoxicity assay demonstrated acceptable toxicity profile of the synthesized nanoparticles up to a concentration of $10 \mu \mathrm{g} / \mathrm{ml}$. Intravenously administered CS-DX-SPIONs in orthotopic C6 gliomas in rats accumulated in the tumor site as shown by high-resolution MRI (11.0 T). Retention of nanoparticles resulted in a significant contrast enhancement of the tumor image that was accompanied with a dramatic drop in $T_{2}$ values $(P<0.001)$. Subsequent histological studies proved the accumulation of the nanoparticles inside glioblastoma cells.

Conclusion: Hybrid chitosan-dextran magnetic particles demonstrated high MR contrast enhancing properties for the delineation of the brain tumor. Due to a significant retention of the particles in the tumor an application of the CS-DX-SPIONs could not only improve the tumor imaging but also could allow a targeted delivery of chemotherapeutic agents.

Keywords: brain tumor, glioblastoma, tumor targeting, chitosan, superparamagnetic iron oxide nanoparticles, magnetic resonance imaging

\section{Introduction}

Despite multimodal treatment approach for glioblastoma multiforme that currently includes surgical resection of the tumor with radio- and pharmacotherapy with temozolomide (TMZ), the survival of patients does not exceed 15 months. ${ }^{1,2}$ Introduction of the tumor-treating fields (TTFields) as locoregional treatment only slightly improved 
the median overall survival in subgroups of patients, up to 20.5 months. ${ }^{3}$ These data indicate the necessity for novel diagnostic and therapeutic modalities in neuro-oncology. Presumably, theranostic approaches could be based on the chitosan nanoparticles that show a high biocompatibility at relatively low costs. Previously, several studies reported the application of chitosan particles as nanocarriers of antiglioma agents. ${ }^{4-10}$ Thus, Liu et $\mathrm{al}^{4}$ showed that chitosan surface-modified poly(lactideco-glycolide)-chitosan nanoparticles (PLGA/CS NPs) loaded with carmustine (bis-chloroethylnitrosourea, BCNU) and its sensitizer ( $\mathrm{O}^{6}$-benzylguanine, $\mathrm{BG}$ ) had a strong antitumor effect in the F98 glioma-bearing mice model. In another study by Lollo et al, ${ }^{11}$ the authors could demonstrate similar effects with paclitaxel (PTX)-loaded particles in GL261 gliomabearing mice. Subsequent functionalization of the chitosan nanoparticle surface with bioligands further enhanced the ability of the particles to cross the blood-brain barrier (BBB) and their brain tumor accumulation level. Up-to-date, various ligands were proposed for decoration of the surface, including iRGD (tumor targeting peptide [CRGDKGPDC]) with high affinity to $\alpha v$ integrins, ${ }^{4}$ transferrin receptor targeting agents, ${ }^{5}$ and lactoferrin. ${ }^{12}$

Alternative strategies based on chitosan particles could be their application, not only for the delivery of antitumor agents, but also for the detection of glioma. For the imaging of the glioblastoma in the current work, dextran-coated superparamagnetic iron oxide nanoparticles (DX-SPIONs) that were further coupled with chitosan were produced. The coupling of chitosan to preliminary activated dextran magnetic nanoparticles (MNPs) can increase the surface charge of the latter and thus improve the tumor uptake of SPIONs. Hence, as shown by Barrow et al, ${ }^{13}$ an increase in charge of the $40 \mathrm{kDa}$ dextran-based iron oxide nanoparticles (IONPs) from -1.5 to $+18.2 \mathrm{mV}$ provided a sixfold increase in uptake in mouse mesenchymal stem cells (MSCs) with respect to neutral dextran-coated particles. Furthermore, the hybrid chitosan-dextran-coated nanoparticles exert stronger membrane interactions as compared to monodextran particles. ${ }^{14}$ Due to their unique magnetic and optical characteristics, such as high paramagnetism, low magnetic coercivity, and high Curie temperature, SPIONs show favorable characteristics compared to other types of magnetic nanocarriers. ${ }^{15}$ Magnetic properties of the SPIONs were also employed for the development of the contrast agents for the magnetic resonance imaging (MRI) of brain tumors. ${ }^{16,17}$

In our study, the tumor targeting ability and the MRI contrast-enhancing properties of the synthesized hybrid dextran-chitosan SPIONs (CS-DX-SPIONs) were assessed in an orthotopic C6 Wistar rat model. In this study, we report that chitosan-based MNPs could be successfully employed for the MRI detection of brain tumors.

\section{Materials and methods Synthesis of the DX-SPIONs}

The superparamagnetic (SPM) nanoparticles were obtained from iron salt solutions by coprecipitation at $80^{\circ} \mathrm{C}$ in alkaline media, as described previously. ${ }^{18}$ Briefly, $\mathrm{FeSO}_{4}$ and $\mathrm{FeCl}_{3}$ $\left(\mathrm{Fe}^{2+} / \mathrm{Fe}^{3+}\right.$ ratio of 1:2) salts were dissolved together with $\mathrm{CsCl}$ in water. ${ }^{19}$ The magnetite precipitation was induced in an inert atmosphere by titration with $\mathrm{NH}_{4} \mathrm{OH}$ solution and collected using a permanent magnet. The nanoparticle suspension was treated by ultrasound at $22 \mathrm{kHz}$ for 15 minutes. To prevent the sedimentation of the nanoparticles, $10 \mathrm{kDa}$ molecular weight (MW) dextran (Sigma-Aldrich Co., St Louis, MO, USA) was added into dispersion in the process of ultrasound application. The obtained SPION stock solution was washed and separated into fractions by centrifugation and microfiltration using $0.2 \mu \mathrm{m}$ pore size membranes (EMD Millipore, Billerica, MA, USA). The concentration of Fe in the suspension was assessed by ultraviolet (UV) absorption (at $\lambda=480 \mathrm{~nm}$ ) of the thiocyanate-Fe(+3) complex.

\section{Preparation of the hybrid chitosan nanocarriers (CS-DX-SPIONs)}

About 94\% deacetylated chemical-grade chitosan (MW $30 \mathrm{kDa}$ ) was prepared from crab shells in formate solution at $\mathrm{pH}$ 6-7.4. DX-SPIONs were transferred into glass and aminated with epichlorohydrin. Chitosan was dissolved in $100 \mathrm{~mL}$ of water under magnetic stirring at $\mathrm{pH}$ 7.0. Hybrid nanoparticles were prepared by ionotropic gelation by mutual interaction of chitosan and dextran with negatively charged ion tripolyphosphate (TPP) (Vekton, St. Petersburg, Russia). An aqueous solution of TPP was added dropwise to the chitosan solution with suspended SPIONs. The hybrid nanoparticles were formed instantly after addition of TPP. The tertiary composition in reaction medium was as follows: polyvinylpyrrolidone (MW 3.6 MD; BASF), polyvinylpyrrolidone (PVP) (3\% g/mL), TPP $(0.04 \% \mathrm{~g} / \mathrm{mL})$, and chitosan $(0.25 \% \mathrm{~g} / \mathrm{mL})$.

\section{Physicochemical characterization of CS-DX-SPIONs}

The particle size of SPIONs and size distribution were analyzed by transmission electron microscopy (TEM) using the JEOL-2000 microscope (JEOL, Tokyo, Japan) and dynamic light scattering (DLS) with the help of Malvern Instruments (Malvern, UK). Electrophoretic properties and hydrodynamic size were measured on Zetasizer Nano (Malvern Instruments). The relaxation times $T_{1}, T_{2}$, and $T_{2}{ }^{*}$ 
and ${ }^{1} \mathrm{H}$ nuclear magnetic resonance (NMR) spectra were measured with the NMR spectrometer CXP-300 (Bruker Optik GmbH, Ettlingen, Germany) at the magnetic field of $7.1 \mathrm{~T}$ (corresponding to $300 \mathrm{MHz}$ for ${ }^{1} \mathrm{H}$ ). The NMR spectra were registered with a one-pulse sequence by applying Fourier transform of free induction decay. The $T_{2}{ }^{*}$ values were estimated from line width at half height. To assess the $T_{1}$ and $T_{2}$ relaxation times, the inversion recovery and Carr-PurcellMeiboom-Gill (CPMG) pulse sequences were employed. Proton relaxation times in buffer solution were recorded as a function of magnetic conjugate concentration. The $R_{1}, R_{2}$, and $R_{2}^{*}$ (relaxivity) coefficients of relaxation efficiency were defined from the slopes of concentration plots. The ${ }^{31} \mathrm{P}$ NMR spectra were obtained on a Bruker CXP-300 at $121 \mathrm{MHz}$ using a $5 \mathrm{~mm}$ phosphorus probe and at $162 \mathrm{MHz}$ on a WM 400 spectrometer using a $10 \mathrm{~mm}$ broadband probe. Phosphorus chemical shifts are referred relative to $\mathrm{H}_{3} \mathrm{PO}_{4}$ solution at $\mathrm{pH} 2$. The ${ }^{31} \mathrm{P}$ spin-lattice relaxation rate constants were obtained from 10 to 12 relaxation intervals using the inversion recovery pulse sequence at $120 \mathrm{MHz}$ without proton decoupling.

\section{Magnetic characterization of CS-DX-SPIONs}

To characterize the magnetic state of the CS-DX-SPIONs (single-/multi-domain) and to estimate their average magnetic moment, we employed our home-built setup, registering the nonlinear longitudinal response (NLR) of MNPs to a weak ac magnetic field $h(t)=h \sin (\omega t)$ parallel to steady field $H$ (the frequency $f=\omega / 2 \pi=15.7 \mathrm{MHz}$ and $h=13.8 \mathrm{Oe}$ ). The setup included two coaxial Helmholtz coils (providing the linear scanning of steady field $H$ with frequency, $F_{\text {sc }}$, varied in the range $8-10^{-2} \mathrm{~Hz}$ ) combined with the electromagnet (for the creation of a constant field $H_{0}$ if it is necessary) and a transmitter-receiver high frequency coil of a two-mode resonant system. The resonant system, on the one hand, creates $a c$ field on a sample under study and, selects from the NLR the second harmonic $M_{2}$ of the magnetization (NLR- $\left.M_{2}\right)$ generated by a sample. ${ }^{20}$ The $M_{2}$ signal components, ie, $\operatorname{Re} M_{2}(H)$ and $\operatorname{Im} M_{2}(H)$, are recorded simultaneously as functions of $H$. The frequency of $H$-scan, $F_{\text {sc }}$, can be changed within 8-0.02 Hz, which allows the testing of the state (single-/ multi-domain) of MNPs. Indeed, in the multi-domain state, the magnetization reversal is determined by movement of domain boundaries, which is fast, and $H$-hysteresis will not depend on $F_{\text {sc }}$. However, in single-domain MNPs, the field hysteresis appears in the blocking regime below blocking temperature, $T_{\mathrm{b}}$. The latter can be found from condition $\tau=$ $\tau_{\text {mes }}$ (where $\tau$ is the time of the magnetic relaxation of MNPs ensemble and $\tau_{\text {mes }}$ is the time of measurements), and in our experiments, it depends on $F_{\mathrm{sc}}$. Indeed, in the case of the uniaxial anisotropy applicable to our MNPs, $1 / \tau=f_{0} \exp \left(-E_{\mathrm{b}} / k T\right)$ (where anisotropy energy $E_{\mathrm{b}}=K V, k$ is the Boltzmann constant, $K$ is the anisotropy constant, $V$ is an average volume of a particle, and the frequency $f_{0}$ is of the order of $10^{9} \mathrm{~s}^{-1}$ ) and $T_{\mathrm{b}}=K V /\left[k \cdot \ln \left(f_{0} / F_{\mathrm{sc}}\right)\right] \cdot{ }^{21}$ The dependence of $H$-hysteresis on $F_{\text {sc }}$ evidences the single-domain state of MNPs in our study. Usually, NLR- $M_{2}$ measurements are carried out under the condition $M_{2} \propto h^{2}$, which allows the application of the perturbation theory for analysis of the results. The main contribution to $\operatorname{Re} M_{2}(H)$ arises due to the nonlinear behavior of $M(H)$, so in the regime of the MNP ensemble close to the SPM one (a weak $H$-hysteresis/anhysteretic of $M_{2}(H)$ response), the $\operatorname{Re} M_{2}(H)$ dependence can be semiquantitatively described by $\partial^{2} L(H) / \partial H^{2}+k_{\mathrm{PM}} H$, where $L(H)$ is the Langevin function and the term $k_{\mathrm{PM}} H$ takes into account possible small linear contributions from low-molecular paramagnetic fraction. ${ }^{22}$ The $\operatorname{Re} M_{2}(H)$ reaches extreme values near the inflection point of $M(H)$, which is dependent on the average moment of MNPs that allows to estimate the latter. This description is not quantitative since it does not take into account the effect of the magnetic field on relaxation processes. This could be done by utilizing the Gilbert-Landau-Lifshitz formalism, but this is out of the scope of our study. ${ }^{23}$

\section{Assessment of cellular interactions of CS-DX-SPIONs Cells}

All employed cell lines (rat C6 glioma, human U87 glioma, and human cervix carcinoma HeLa cells) were obtained from the Russian Cell Culture Collection of the Institute of Cytology of the Russian Academy of Sciences (St Petersburg, Russia). $\mathrm{C} 6$ and $\mathrm{HeLa}$ cells were cultured in a $\mathrm{CO}_{2}$ incubator (at $37^{\circ} \mathrm{C}, 6 \% \mathrm{CO}_{2}$ ) in DMEM culture medium supplemented with $2 \mathrm{mM}$ L-glutamine, 10\% fetal bovine serum (FBS), and antibiotics (ie, streptomycin [100 units $/ \mathrm{mL}$ ] and penicillin $\mathrm{G}$ $[100 \mu \mathrm{g} / \mathrm{mL}])$. Human U87 glioma cells were harvested in complete RPMI-1640 culture medium (Sigma-Aldrich Co.) supplemented with antibiotics and 10\% FBS.

\section{Cytotoxicity assay}

For the analysis of cytotoxicity of CS-DX-SPIONs, the commercial non-radioactive cytotoxicity assay kit, CytoTox $96^{\circledR}$ (Promega Corporation, Fitchburg, WI, USA), was employed in accordance with the manufacturer's protocol. Briefly, tumor cell lines (ie, C6 glioma, U87, and HeLa cells) were cocultured (for 1, 3, 12, and 24 hours) with SPIONs or hybrid CS-DX-SPIONs taken at different Fe concentrations (of 1, $5,10,50$, and $150 \mu \mathrm{g} / \mathrm{mL}$ ). The obtained supernatants from 
the samples were analyzed for the concentration of lactate dehydrogenase (LDH).

\section{Confocal microscopy}

For the assessment of cellular interactions of synthesized nanoparticles with tumor cells (ie, C6 glioma, HeLa cells, and U87 cells $\left[1 \times 10^{6}\right.$ cells $\left.\left./ \mathrm{mL}\right]\right)$, the latter were permitted to settle on poly-L-lysine-coated glass slides. SPIONs or CS-DX-SPIONs (Fe concentration: $150 \mu \mathrm{g} / \mathrm{mL}$ ) were coincubated with the tumor cells for $1,3,12$, and 24 hours in a $\mathrm{CO}_{2}$ incubator $\left(37^{\circ} \mathrm{C}, 6 \%\right.$ $\mathrm{CO}_{2}$ ). Prior to cell imaging, the nuclei of the cells were stained with 4,6-diamidine-2-phenylindole (DAPI) solution (Vector Laboratories, Burlingame, CA, USA). A series of the fluorescence $z$-images were recorded using a Leica TCS SP 5 confocal microscope (Leica Microsystems, Heidelberg, Germany).

\section{Flow cytometry analysis}

For assessment of the nanoparticle internalization by tumor cells, a flow cytometer equipped with a coherent red solid state diode (635 nm), uniphase argon laser (488 nm), side scatter detector (SSD), and forward scatter detector (FSD), Cytomics FC500 (Beckman Coulter, Indianapolis, IN, USA) was used. The setups of the cytometer measured forward scattering (FSC) and side scattering (SSC) linearly and logarithmically. After an incubation period of 24 hours of the tumor cells (C6, U87, and HeLa) with DX-SPIONs, CS-DX-SPIONs (Fe concentration of $150 \mu \mathrm{g} / \mathrm{mL}$ ), or phosphate buffered saline (PBS), the cells were analyzed by flow cytometry. The scatter parameters derived from the gated scatter region were used for counting cells within a large SSC (log) versus FSC (linear) histogram.

\section{Gel-phantom model}

For the assessment of the magnetic properties of the CSDX-SPIONs following cellular uptake, the C6 glioma cells were coincubated with DX-SPIONs (Fe concentration: $150 \mu \mathrm{g} / \mathrm{mL}$ ), CS-DX-SPIONs (Fe concentration: $150 \mu \mathrm{g} / \mathrm{mL}$ ), or PBS for 24 hours. Following coincubation and washing with PBS, the cells were embedded into $1 \%$ agarose gel. For the assessment of the intracellular nanoparticle localization, several anatomical high-resolution turbo spin echo (SE) $T_{2}$-weighted coronal images ( 20 slices in total) were obtained using the following parameters: repetition time (TR)/echo time (TE) 4,200/36 ms, flip angle (FA) $180^{\circ}$, slice thickness of $1.0 \mathrm{~mm}$, interslice distance $1.2 \mathrm{~mm}$, field of vision (FOV) $3.0 \times 3.0 \mathrm{~cm}$, and matrix $256 \times 256$. Subsequently, gradient echo (GE) scans (FLASH) (TR/TE 350/5.4 ms, FA 40 , slice thickness $1.0 \mathrm{~mm}, 3.0 \times 3.0 \mathrm{~cm}$, and matrix $256 \times 256$ ) and SE $T_{1}$-weighted images (TR/TE $1,500 / 7.5 \mathrm{~ms}, \mathrm{FA} 180^{\circ}$, slice thickness $1.0 \mathrm{~mm}$, FOV $3.0 \times 3.0 \mathrm{~cm}$, and matrix $256 \times 256$ ) were obtained in the coronal plane. Accumulation of the DX-SPIONs or CS-DX-SPIONs in the samples was assessed using multi-scan multi-echo (MSME) sequences.

\section{Orthotopic C6 glioma model}

Male Wistar rats with a body weight of $280-300 \mathrm{~g}$ were obtained from the "Rappolovo" RAMN animal nursery (St Petersburg, Russia). For all animal experiments, the approval has been provided by the local ethical committee of First Pavlov Saint Petersburg State Medical University (St Petersburg, Russia) in accordance with institutional guidelines for the welfare of animals. Before mounting in a stereotactic frame (David Kopf Instruments, Tujunga, CA, USA), the rats were anesthetized with $0.2 \mathrm{~mL} \%$ Rometar (xylazine hydrochloride; "Bioveta", Ivanovice na Hané, Czech Republic) and $10 \mathrm{mg}$ tiletamine hydrochloride and zolazepam ("Virbac santé Animale", Carros, France) intraperitoneally. C6 rat glioma cells $\left(1 \times 10^{6}\right.$ cells in $10 \mu \mathrm{L}$ of PBS $)$ were stereotactically infused into the nucl. caudatus dexter (right caudate nucleus) using a Hamilton microsyringe.

\section{Magnetic resonance assessment of CS- DX-SPIONs' tumor-targeting potential}

On the 15th day following the intracranial injection of the C6 tumor cells for the assessment of the accumulation of DX-SPIONs or CS-DX-SPIONs in the tumor site, the animals were randomly divided into three groups as follows (six rats in each group): 1) control group - intravenous (i.v.) injection of PBS via the tail vein; 2) i.v. administration of DX-SPIONs (Fe concentration of $2.5 \mathrm{mg} / \mathrm{kg}$ ); and 3) i.v. administration of the CS-DX-SPIONs (Fe concentration of $2.5 \mathrm{mg} / \mathrm{kg}$ ). Twenty-four hours after i.v. injection, intratumoral retention of MNPs was assessed using a high-field MRI scanner (11.0 T) (Avance III $400 \mathrm{WB}$; Bruker Optik GmbH) in the following conventional sequences: 1) $T_{1}$-weighted fast-field echoes (TR/TE [TR] 1,500/7.5 ms, FOV $3.0 \times 3.0 \mathrm{~cm}$, matrix $256 \times 256, \mathrm{FA} 180^{\circ}$ number of signals averaged [NSA]); 2) $T_{2}$-weighted turbo $\mathrm{SE}\left(\mathrm{FA} 180^{\circ}, \mathrm{TR} 4,200 \mathrm{~ms}\right.$, and $\mathrm{TE}$ $36 \mathrm{~ms}$, FOV $3.0 \times 3.0 \mathrm{~cm}$, matrix $256 \times 256$ ); 3) FLASH scans (GE) (FA 40 ${ }^{\circ}$, TR $350 \mathrm{~ms}$, and TE $5.4 \mathrm{~ms}$ ); and 4) MSME regimen. All images were obtained with a slice thickness of $1.0 \mathrm{~mm}$ in the coronal plane. Assessment of the MRI scans was carried out using the Paravision 3.1 software package (Bruker BioSpin GmbH, Rheinstetten, Germany) and Analyze software (AnalyzeDirect, Inc., Overland Park, KS, USA).

\section{Histology}

For histological analysis, the rats were sacrificed using an intraperitoneal injection of pentobarbital (150-200 mg/kg) 
with subsequent perfusion with PBS (100 mL) and 4\% paraformaldehyde (PFA). For the analysis of the intratumoral distribution of nanoparticles, the obtained serial cryosections (5-7 $\mu \mathrm{m})$ were additionally stained with DAPI and assessed by using a Leica TCS SP5 confocal microscope in a reflecting laser scanning regimen (laser $488 \mathrm{~nm}$ [Ar/Kr]).

\section{Statistical analysis}

For the analysis of the statistical differences, one-way analysis was carried out by the Kruskal-Wallis test. Student's $t$-test was performed to compare the obtained animal data using Statistica Version 9.2 program. Differences were considered to be statistically significant when $P<0.05$.

\section{Results}

\section{Synthesized CS-DX-SPIONs exhibit high-contrast-enhancing properties}

The sequence of the steps in the synthesis of hybrid SPIONs is represented in Figure 1. Following the addition of dextran to the prepared SPIONs for stabilization of the particles, the average size ranged between 30 and $40 \mathrm{~nm}$. According to TEM measurements, DX-SPIONs are presented as round-shaped particles. For further coating of the particles with chitosan, the surface of DXSPIONs was activated by $\mathrm{NH}_{2}$ groups through addition of epichlorohydrin. Chemical activation did not change the stability of nanoparticle dispersion (as assessed by DLS). Size distribution measured by the DLS method was $55 \mathrm{~nm}$. The hybrid dextran-chitosan coat of the magnetite core was obtained in the cross-linking procedure with the help of TPP shown in Figure 1 as Step III. Negatively charged ions of TPP induce ionotropic gelation of chitosan and dextran. To study the molecular mechanism of interaction between TPP and chitosan, ${ }^{31} \mathrm{P}$ NMR measurements were conducted with gradual increase in TPP concentration in the reaction solution. According to the ${ }^{31} \mathrm{P}$ NMR spectra, three $\alpha-, \beta$-, and $\gamma-\mathrm{PO}_{4}$ groups produce two well-resolved lines at different chemical shifts (Figure 2). The low-shift

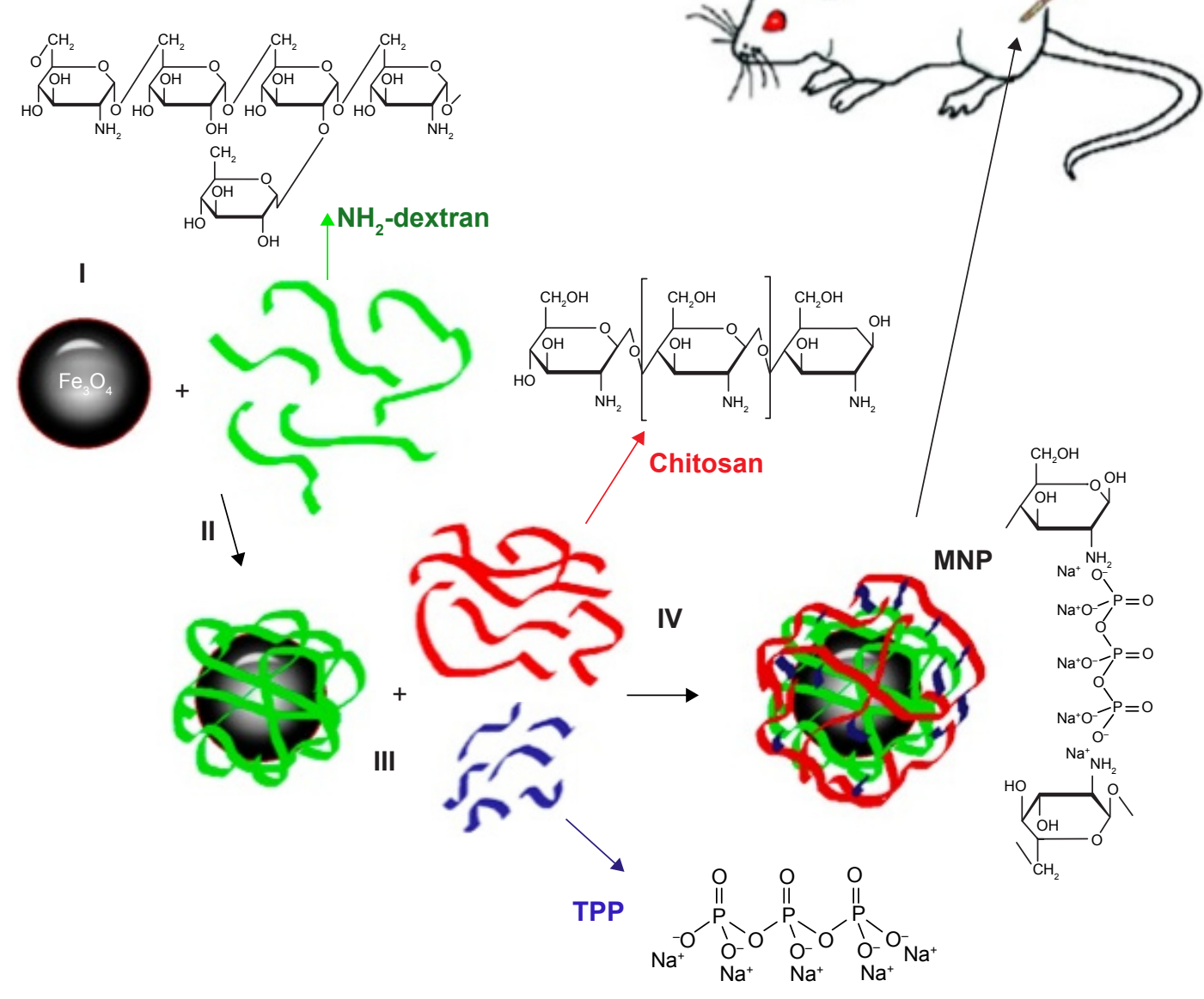

Figure I Schematic representation of the synthesis of chitosan-based superparamagnetic iron oxide nanoparticles (CS-DX-SPIONs).

Abbreviations: CS-DX-SPIONs, chitosan-dextran superparamagnetic iron oxide nanoparticles; MNP, magnetic nanoparticle; TPP, tripolyphosphate. 


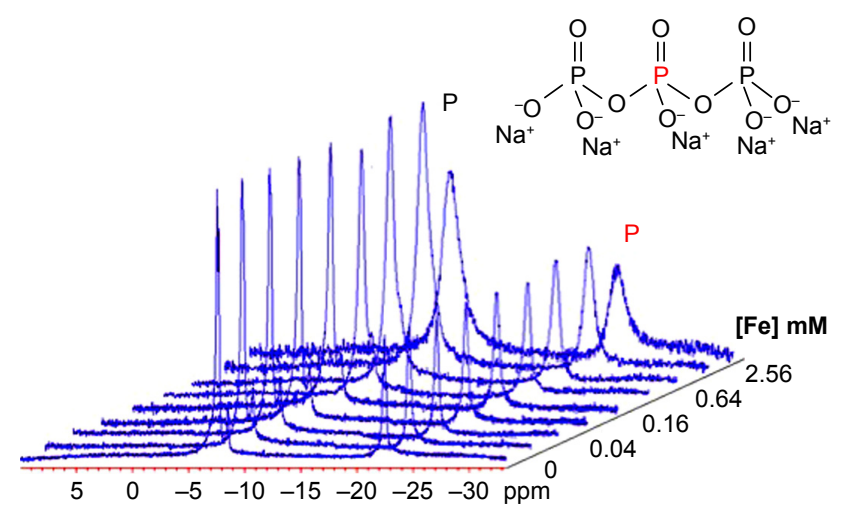

Figure $2{ }^{31}$ Phosphorus NMR spectroscopy of the TPP.

Abbreviations: NMR, nuclear magnetic resonance; TPP, tripolyphosphate.

line (ppm) belongs to $\alpha$ and $\gamma$ phosphate groups. In the process of titration, initial addition of TPP did not lead to the corresponding increase in intensity resonance. According to the theoretical analysis, the ${ }^{31} \mathrm{P}$ relaxation rates of phosphate groups in strong magnetic field (7.1 T) due to dipole-dipole (DD) and chemical shift anisotropy (CSA) mechanisms are well described by Bloembergen et al. ${ }^{24,25}$ In line with this theory, ion-ion interaction of $\mathrm{PO}_{4}$ groups with protonated $\mathrm{NH}_{3}^{+}$groups reduces molecular motion of TPP and leads to strong decrease in magnetic relaxation times $T_{1}$ and $T_{2}$ and thus loss of spectral resolution. At low TPP concentrations, the resonance lines are invisible. At saturation threshold of all free $\mathrm{NH}_{3}^{+}$groups, excess of TPP agent gives rise to narrow $\mathrm{PO}_{4}$ lines in spectra with intensity proportional to TPP concentration. The observed concentration dependencies are in good agreement with the hypothesis that one molecule of TPP binds $2-3$ positively charged $\mathrm{NH}_{3}$ groups. Coverage of magnetic nuclei by the dextran-chitosan coat results in an increase in the hydrodynamic diameter of nanoparticles and initiation of magnetic moment in the whole nanostructure. With respect to the small size of the nanoparticles $(40 \mathrm{~nm})$ one might assume that the magnetic particle consists of a single domain nanocrystal. In this case, the nanoparticle would behave as an SPM unit exposing classical hysteresis behavior in accordance to the Langevin law. ${ }^{26}$ Otherwise, the particles behave as a ferromagnetic material. To elucidate the magnetic nature of hybrid CS-DX-SPIONs, the novel NLR- $M_{2}$ approach was applied to assess the particles for hysteresis criteria. Both components, $\operatorname{Re} M_{2}$ and $\operatorname{Im} M_{2}$ of nonlinear response, were measured versus steady field $H$ at $T=294 \mathrm{~K}$ at different rates of field scan, $F_{\text {sc }}$. The obtained $H$-dependencies display the characteristic signals, exhibiting extremes at a weak magnetic field $H$ in $H>0$ range of $H$-scan, opposite signs in $\operatorname{Re} M_{2}(H)$ and $\operatorname{Im} M_{2}(H)$ components, and the weak field hysteresis, which decreases with a reduction in $F_{\mathrm{sc}}$. These features correspond to $M_{2}$ response of ensemble of MNPs in the single domain state (Figure 3). ${ }^{27} \mathrm{~A}$ weak field hysteresis is predictive for a close to SPM regime, which is in accordance with a single domain structure. $M_{2}$ responses yielded by the $\mathrm{Fe}_{3} \mathrm{O}_{4}$ nuclei and magnetic parameters of the latter in the first approximation are not affected by the formation of the hybrid dextran-chitosan shell. The response of an ensemble of NPs in SPM regime is characterized by the absence of field hysteresis. It is obvious that in this case the magnetization $M$ will be directed along the field, parallel to the $z$ axis. As for complex magnetic materials such as manganites, cobaltites, and composite substances on co-compounds, the shape of hysteresis curves depends on $F_{\text {sc }}$ and temperature, and SPM regime occurs above blocking temperature $T_{\mathrm{B}}{ }^{28,29}$ The hybrid CS-DX-SPIONs have high magnetic moment to accelerate magnetic relaxation of aqueous protons in suspension due to large perturbation of the magnetic field in the neighborhood of MNPs. Inhomogeneous magnetic fields in the probe can be calculated as the sum of dipole fields induced by magnetite cores. The inhomogeneous

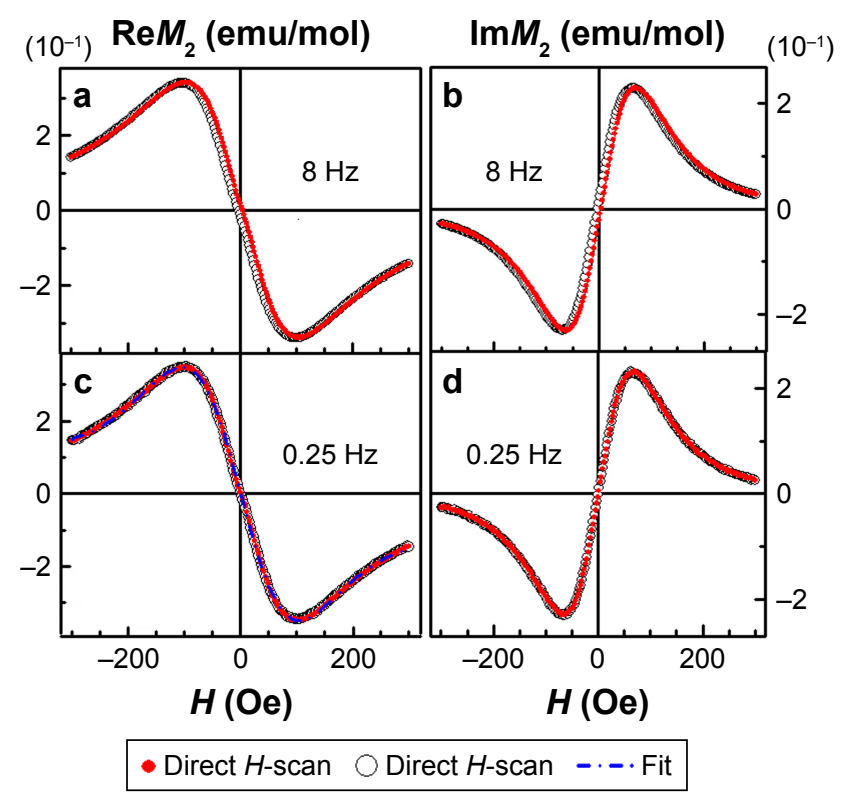

Figure 3 Second harmonic measurements of CS-DX-SPIONs in water at different frequencies of $H$-scan, $F_{\text {sc }}=8$ (panels (a) and (b)) and $0.25 \mathrm{~Hz}$ (panels (c) and (d)), $T=294 \mathrm{~K}$.

Notes: Solid and open circles display direct and reverse $H$-scans, respectively. Dashed-dot curve in panel (c) presents fit $\operatorname{Re} M_{2}(H)$ dependence obtained at $F_{\mathrm{sc}}=0.25 \mathrm{~Hz}$ by $\partial^{2} L(H) / \partial H^{2}+k_{\mathrm{pM}} H$.

Abbreviation: CS-DX-SPIONs, chitosan-dextran superparamagnetic iron oxide nanoparticles. 
character of the field shortens the magnetic relaxation times of $\mathrm{H}_{2} \mathrm{O}$.

\section{Assessment of cellular interactions of CS-DX-SPIONs}

Uptake of the synthesized DX-SPIONs and hybrid CS-DXSPIONs was analyzed in tumor cells (C6, U87, and HeLa) following coincubation of the latter with DX-SPIONs or CS-DX-SPIONs at various concentrations of $\mathrm{Fe}^{3+}$ (ie, 1, 10, 50 , and $150 \mu \mathrm{g} / \mathrm{mL}$ ) and incubation periods (ie, 1, 3, 6, 12, and 24 hours). Following an overnight coincubation, the confocal microscopic views show that DX-SPIONs were incorporated into the cytosol of the tumor cells (Figure 4). Magnetic conjugates on the reflective laser scanning are presented as green dots throughout the cytosol of the tumor cells but are not detected in the nucleus of the cells (Figure 4A). In the case of hybrid CS-DX-SPIONs, an increase in the internalization of nanoparticles as compared to DX-SPIONs was detected (Figure 4A). The uptake of the CS-DX-SPIONs was further evaluated as related to their contrast-enhancing properties when coincubated with C6 glioma cells. Accumulation of the hybrid SPIONs in the cells led to the hyperintense $T_{2}$-weighted MRI images and subsequent decrease in $\mathrm{T}_{2}$ values (Figure 4B). This indicates that CS-DX-SPIONs preserve their magnetic contrast-enhancing properties after internalization into cells. Thus, $T_{2}$ values following internalization of the CS-DX-SPIONs constituted $6.0 \pm 1.34 \mathrm{~ms}$ which was significantly lower in comparison to the DX-SPIONs and
A

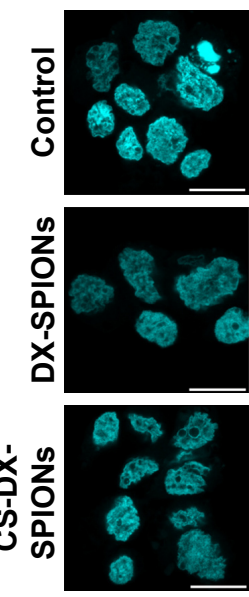

C

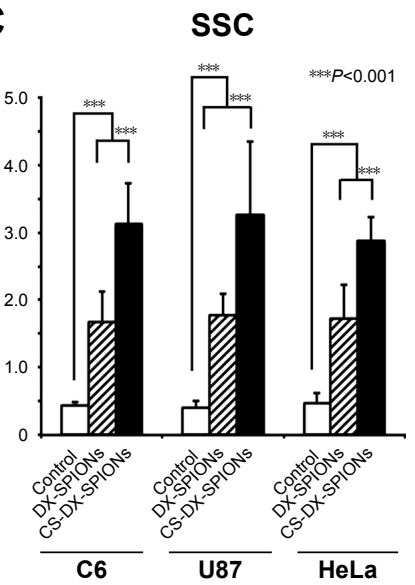

MNPs
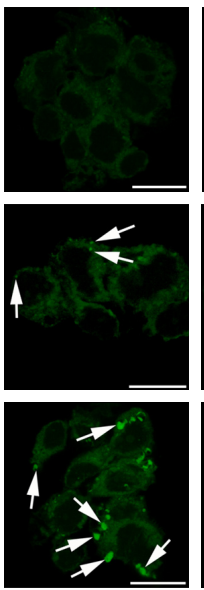

D

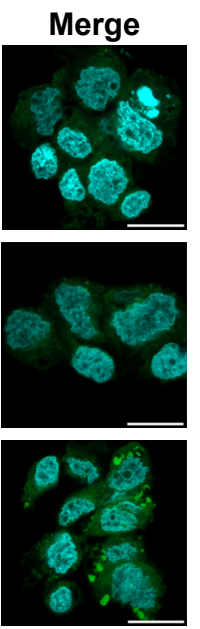

FSC

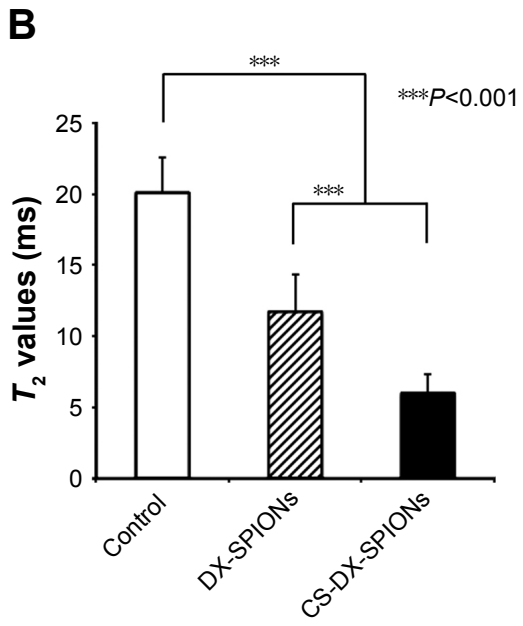

E Cell fluorescence

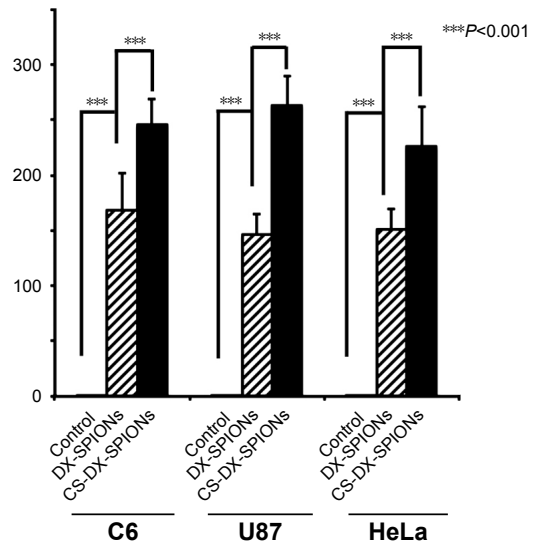

Figure 4 Interaction of CS-DX-SPIONs with cells.

Notes: (A) Confocal microscopy images of the rat C6 glioma cells coincubated for 24 hours with PBS (control), DX-SPIONs, or CS-DX-SPIONs. Nanoparticles were detected using reflecting laser scanning at $488 \mathrm{~nm}$ (presented as green dots). Nuclei were stained with DAPI (blue). Scale bar, $7 \mu \mathrm{m}$. (B) $T_{2}$ values (ms) of the C6 cells following coincubation with PBS (control), DX-SPIONs, or CS-DX-SPIONs for 24 hours in $0.1 \%$ agarose gel. Data are presented as mean \pm SD. (C) SSC, (D) FSC, and (E) cell fluorescence for three cell lines (C6, U87, and HeLa) following coincubation with PBS (control), DX-SPIONs, or CS-DX-SPIONs for 24 hours. The mean and SD were calculated for the three experiments. $T_{2}$ is the spin-spin relaxation time.

Abbreviations: CS-DX-SPIONs, chitosan-dextran superparamagnetic iron oxide nanoparticles; DAPI, 4,6-diamidine-2-phenylindole; DX-SPIONs, dextran-coated superparamagnetic iron oxide nanoparticles; FSC, forward scattering; PBS, phosphate buffered saline; SSC, side scattering. 
control (PBS), 11.72 \pm 2.65 and $20.1 \pm 2.45 \mathrm{~ms}$, respectively $(P<0.001)$ (Figure 4B). To further assess the internalization of nanoparticles, flow cytometry was applied. These data clearly showed an enhanced accumulation of CS-DX-SPIONs in the C6, U87, and HeLa cells as compared to the dextrancoated particles after $24 \mathrm{~h}$ (Figure $4 \mathrm{C}$ and $\mathrm{E}$ ). The retention of the particles significantly increased the granularity (SSC) of the tumor cells, but not their size (FSC) (Figure 4D). After internalization, the cytotoxicity of the nanoparticles, by the detection of LDH in the samples, was analyzed. A dose and time-dependent toxicity of the CS-DX-SPIONs after coincubation with tumor cells was observed (Figure 5). Twentyfour hours after coincubation with CS-DX-SPIONs (at a Fe concentration of $150 \mu \mathrm{g} / \mathrm{mL}$ ), the cytotoxicity of C6, U87, and HeLa cells was $30.1 \% \pm 6.4 \%, 36.2 \% \pm 7.9 \%$, and $27.4 \% \pm 6.2 \%$, respectively.

\section{Targeting of the brain tumor by hybrid CS-DX-SPIONs}

A total of 15 days after orthotopic injection of C6 glioblastoma cells, the animals were randomly divided into three groups (six animals each): 1) control group injected with PBS; 2) i.v. administration of the DX-SPIONs; and 3) i.v. injection of CS-DX-SPIONs. Twenty-four hours after the injection of the nanoparticles, brain tumors were imaged on the high-field MRI scanner. In control animals, the tumor was presented as hypointense on the $T_{1}$-weighted images and hyperintense on $T_{2}$ images (Figure 6). In the
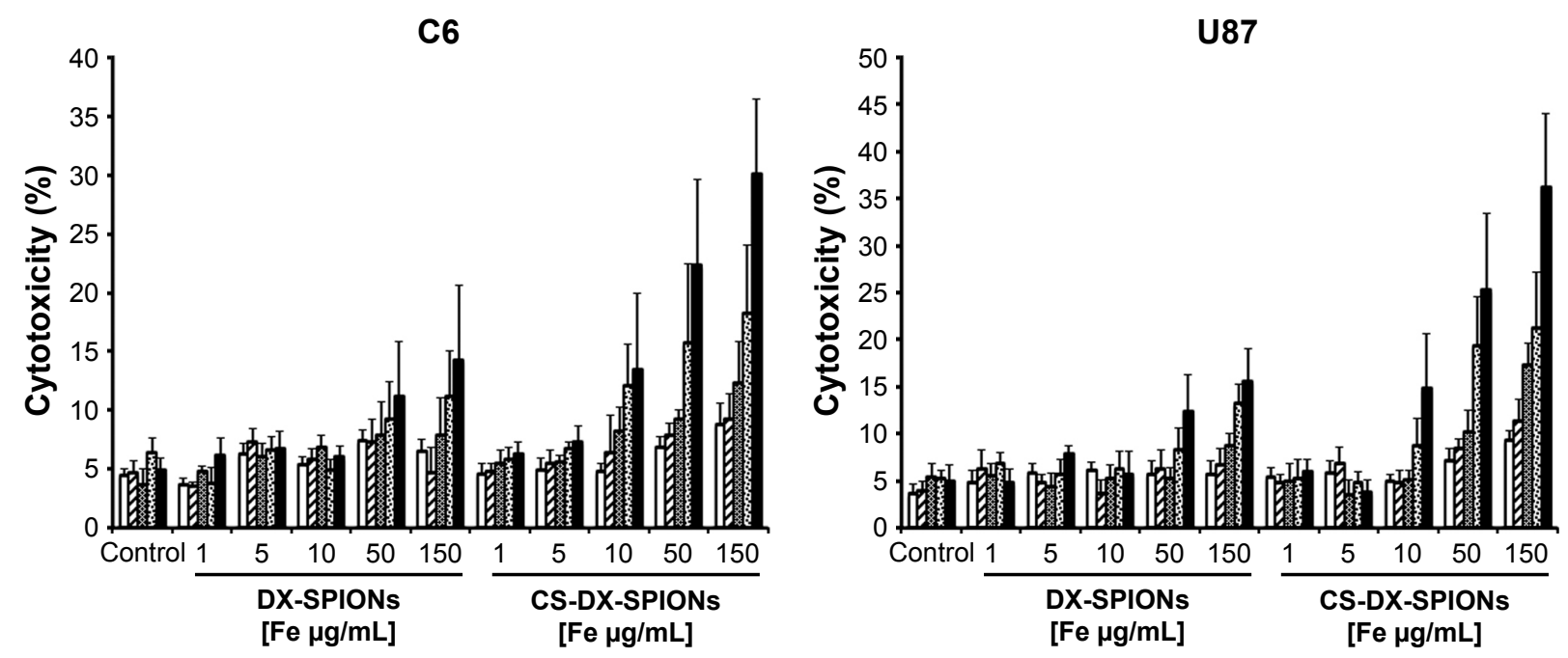

HeLa

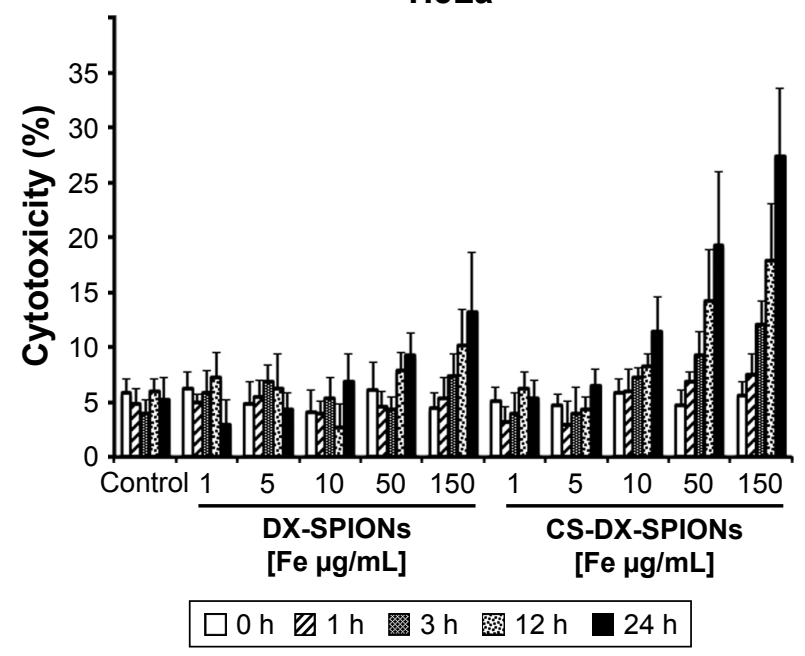

Figure 5 Cytotoxicity assay of the tumor cells (C6, U87, and HeLa) coincubated with PBS (control), DX-SPIONs, or CS-DX-SPIONs. Note: The mean and SD was calculated for the three experiments.

Abbreviations: CS-DX-SPIONs, chitosan-dextran superparamagnetic iron oxide nanoparticles; DX-SPIONs, dextran-coated superparamagnetic iron oxide nanoparticles; h, hour; PBS, phosphate buffered saline. 
A
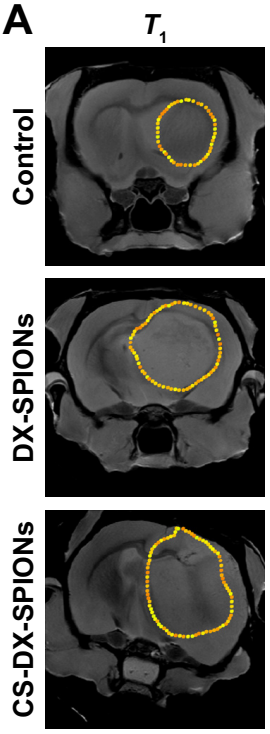

C
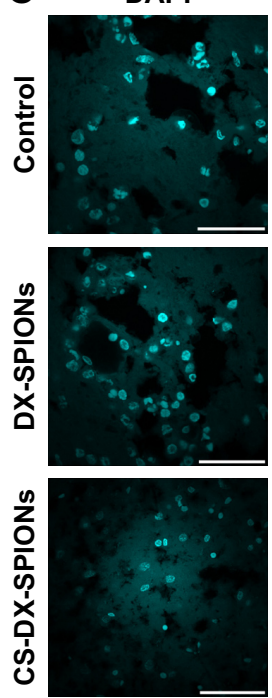

$T_{2}$
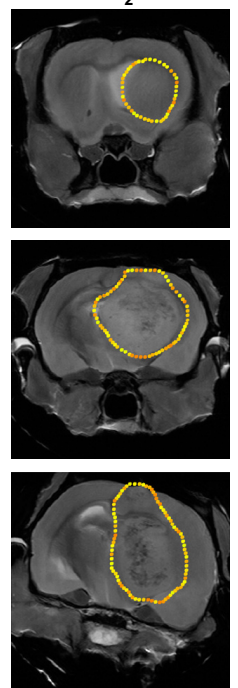

MNPs
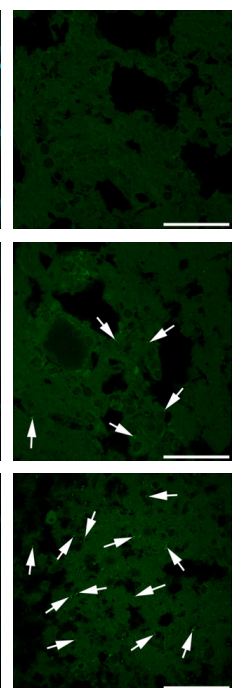

FLASH
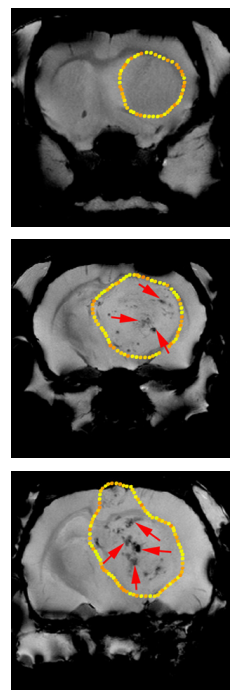

Merge
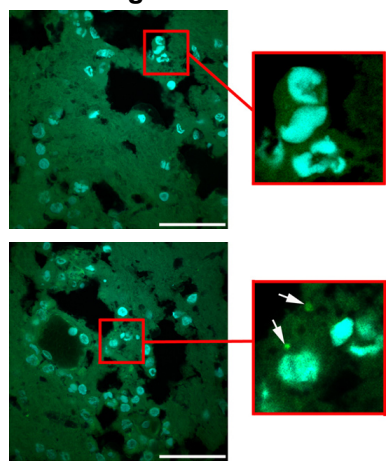

D

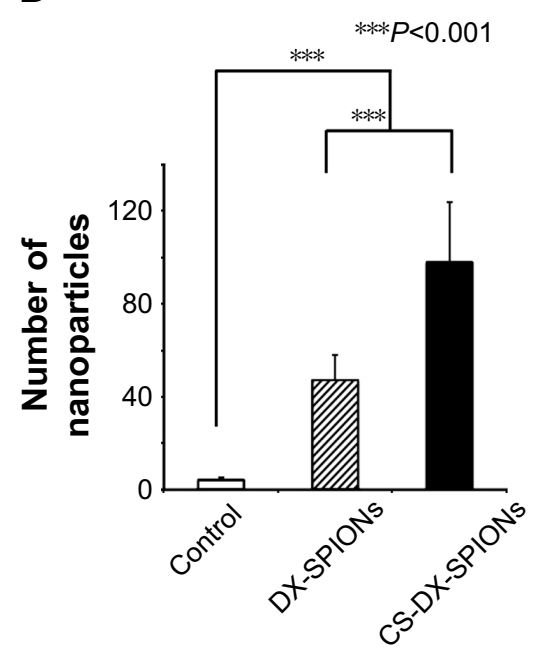

Figure 6 Targeting of the C6 glioma with CS-DX-SPIONs.

Notes: (A) Magnetic resonance images of the brain tumors of the control (PBS), animals treated with DX-SPIONs and CS-DX-SPIONs. $T_{1}$-weighted, $T_{2}$-weighted and gradient echo (FLASH) images of the glioblastoma were obtained. Accumulation of the nanoparticles is shown by solid red arrows. (B) $T_{2}$ values (ms) of the brain tumors in the control (PBS), animals treated with DX-SPIONs and CS-DX-SPIONs. Data are presented as mean \pm SD. (C) Immunofluorescent images of the glioma. Nuclei stained with DAPI (blue). Nanoparticles detected using reflective laser scanning at $488 \mathrm{~nm}$ (green). Scale bar, $40 \mu \mathrm{m}$. (D) Quantification of the nanoparticles in the glioma tissue. Data are presented as mean $\pm \mathrm{SD}$. $T_{1}$ - spin-lattice relaxation time and $T_{2}$-spin-spin relaxation time.

Abbreviations: CS-DX-SPIONs, chitosan-dextran superparamagnetic iron oxide nanoparticles; DAPI, 4,6-diamidine-2-phenylindole; DX-SPIONs, dextran-coated superparamagnetic iron oxide nanoparticles; MNPs, magnetic nanoparticles; PBS, phosphate buffered saline.

DX-SPIONs-injected animal group, glioma was characterized by a hypointense signal in the $T_{2}$-weighted imaging. Twenty-four hours after i.v. infusion of CS-DX-SPIONs, a significant decrease in the $T_{2}$ relaxivity has been observed in comparison to control and SPION-injected animals $(17.1 \pm 1.4$, $10.2 \pm 1.4$, and $5.8 \pm 1.2 \mathrm{~ms}$, respectively; $P<0.001$ ). Reduction of the transverse relaxation time $T_{2}$ is accompanied by the growth of image contrast due to the darkening of accumulation sites of MNPs. Subsequent histological assessment of brain tumor cryosections confirmed the accumulation of the
MNPs inside glioma cells (Figure 6C and D). The distribution pattern of DX-SPIONs or hybrid CS-DX-SPIONs retention in the tumor did not differ.

\section{Discussion}

Nanotechnology can provide a novel theranostic approach in managing glioblastoma multiforme. ${ }^{30,31}$ In our studies, SPIONs with a high MRI contrast enhancement capacity have been used. ${ }^{32}$ For an improved biocompatibility, nanoparticles were coated with chitosan that increased the charge 
of the dextran-coated nanoparticles to $+19.2 \mathrm{mV}$. Chitosan, a polysaccharide with a positive charge at neutral or acidic $\mathrm{pH}$, has advantageous biocompatibility as a carrier material, ${ }^{33}$ a strong permeation enhancing capability for hydrophilic compounds, ${ }^{34}$ and bioadhesive properties. ${ }^{35}$ An increase in charge of the nanoparticles also resulted in an enhanced uptake of CS-DX-SPIONs by tumor cells (Figure 4). The same effect was previously reported for positively charged pullulan-coated NPs that were incorporated by bone marrowderived rat $\mathrm{MSCs}^{36}$ or mouse MSCs. ${ }^{13}$ On the other hand, the decrease in the surface charge abrogated the internalization of the nanoparticles. ${ }^{36}$

Although we observed an enhanced intracellular uptake of the particles, we subsequently determined an increased cytotoxicity of CS-DX-SPIONs (Figure 5). This could be explained by the production of reactive oxygen species (ROS) and subsequent DNA damage. ${ }^{37,38}$ Increased amounts of ROS might also negatively affect mitochondrial DNA (mDNA). ${ }^{39}$

Synthesized CS-DX-SPIONs also act as a negative MRI contrast agent. The measured proton relaxation rates of water in magnetic suspensions of CS-DX-SPIONs clearly indicated a high contrast property of the particles (Figure 3 ). The registered relaxation rates in the hybrid CS-DX-SPIONs resembled the main relaxation features of previously reported dextran-coated MNPs and commercial NP formulations. ${ }^{16,17}$ The composite shell (between chitosan and dextran) crosslinked by TPP does not hamper the diffusion of protons into dephasing volume around MNPs thus preserving the MRI contrast-enhancing properties of the particles. Following coincubation with tumor cells, CS-DX-SPIONs accumulated in cytosol, which resulted in a decrease in $T_{2}$ values when the samples were analyzed using MRI scanning (Figure 4B). This indicates that the obtained NPs preserve their magnetic properties after internalization into cells. In the current study, for the analysis of accumulation of nanoparticles in the glioma, standard MRI sequences (ie, $T_{2}$-weighted scans, GE [FLASH], and multi-scan multi-echo [MSME]) were applied. Recently, other MRI methods for visualization of the MNPs were proposed including investigation of transverse relaxation rates $R_{2}$ and $R_{2}{ }^{*}{ }^{40}$ quantitative susceptibility mapping (QSM), ${ }^{41}$ and susceptibility-weighted imaging (SWI). ${ }^{42-44}$ In the study by Blasiak et al, the highest contrast-to-noise ratio for the detection of the iron deposition in an U87MG glioma model was achieved when the SWI was employed in comparison to any other pulse sequences (ie, SE and GE). ${ }^{42}$ The magnitude of magnetic relaxation rate of hybrid CS-DX-SPIONs corresponded to the contrast enhancement of the agent in MRI scans of brain tumors in rats (Figure 6A).
Following intravenous administration, CS-DX-SPIONs accumulated in glioma cells, which significantly enhanced the contrasts of the tumor. Retention of the particles resulted in a significant decrease in $T_{2}$ values compared to DX-SPIONs.

Accumulation of the particles inside glioma cells could be explained by an enhanced permeation and retention (EPR) effect. ${ }^{45}$ Thus, particles $<100 \mathrm{~nm}$ in diameter extravasate preferentially from the chaotic tumor vasculature, due to the increased permeability of vessels compared to normal tissues. ${ }^{46}$ Presumably, further modifications of the surface of CS-DX-SPIONs with targeting bioligands (eg, antibodies, peptides, proteins, etc) could further increase the accumulation of particles inside glioma tissue. Previously, several studies demonstrated that coating with tumortargeting molecules including anti-Hsp70 antibody, ${ }^{16}$ peptide-targeting interleukin-13 receptor $\alpha 2$ (IL-13R $\alpha 2),{ }^{47}$ anti-VEGF antibodies, ${ }^{48}$ antibodies toward EGFR deletion mutant (EGFRvIII), ${ }^{49}$ anti-insulin-like growth factor binding protein 7 (anti-IGFBP7) single domain antibody, ${ }^{50}$ and many others could show an enhanced particle retention inside tumor cells. ${ }^{51-54}$

\section{Conclusion}

Hybrid CS-DX-SPIONs synthesized by ionotropic gelation at the SPM state have a high MRI contrast-enhancing activity and tumor-targeting potential. In vitro experiments clearly demonstrated that the increase in the surface charge of the nanoparticles due to the chitosan coating subsequently enhanced the intracellular uptake of particles and thus increased their cytotoxic activity. Administration of the magnetic nanocarriers via clinically relevant routes resulted in a significant contrast enhancement of glioma. Diagnostic properties of the synthesized hybrid nanoparticles could be further explored for the development of targeted therapies.

\section{Acknowledgments}

The authors are grateful to Olga G Genbach, Nelly V Koroleva, Olga I Dobrotina, and Prof Dr Oleg V Galibin for help in experiments in vivo on rodents. The MRI measurements were performed in the Center for Magnetic Resonance at St Petersburg State University. The study was supported by the Alexander von Humboldt Fellowship and by the Russian Foundation for Basic Research 15-08-08148A; animal experiments were supported in part by a grant of the Russian Science Foundation 14-50-00068 and by the Federal Agency of Scientific Organizations, Russia, State Grant of the Ministry of Health of the Russian Federation No 32, and by a DFG grant SFB824/3, Germany. 


\section{Disclosure}

The authors report no conflicts of interest in this work.

\section{References}

1. Stupp R, Hegi ME, Mason WP, et al. Effects of radiotherapy with concomitant and adjuvant temozolomide versus radiotherapy alone on survival in glioblastoma in a randomised phase III study: 5 -year analysis of the EORTC-NCIC trial. Lancet Oncol. 2009;10(5):459-466.

2. Weller M, van den Bent M, Tonn JC, et al. European Association for Neuro-Oncology (EANO) guideline on the diagnosis and treatment of adult astrocytic and oligodendroglial gliomas. Lancet Oncol. 2017; 18(6):e315-e329.

3. Stupp R, Taillibert S, Kanner AA, et al. Maintenance therapy with tumor-treating fields plus temozolomide vs temozolomide alone for glioblastoma: a randomized clinical trial. JAMA. 2015;314:2535-2543.

4. Liu C, Yao S, Li X, Wang F, Jiang Y. iRGD-mediated core-shell nanoparticles loading carmustine and $\mathrm{O}(6)$-benzylguanine for glioma therapy. J Drug Target. 2017;25:235-246.

5. Agrawal P, Singh RP, Sonali, et al. TPGS-chitosan cross-linked targeted nanoparticles for effective brain cancer therapy. Mater Sci Eng CMater Biol Appl. 2017;74:167-176.

6. Xu Y, Asghar S, Yang L, et al. Nanoparticles based on chitosan hydrochloride/hyaluronic acid/PEG containing curcumin: in vitro evaluation and pharmacokinetics in rats. Int J Biol Macromol. 2017; 102:1083-1091.

7. Chen Y, Feng S, Liu W, Yuan Z, Yin P, Gao F. Vitamin E succinategrafted-chitosan oligosaccharide/RGD-conjugated TPGS mixed micelles loaded with paclitaxel for U87MG tumor therapy. Mol Pharm. 2017;14:1190-1203.

8. Kievit FM, Stephen ZR, Wang K, et al. Nanoparticle mediated silencing of DNA repair sensitizes pediatric brain tumor cells to $\gamma$-irradiation. Mol Oncol. 2015;9:1071-1080.

9. Danhier F, Messaoudi K, Lemaire L, Benoit JP, Lagarce F. Combined anti-Galectin-1 and anti-EGFR siRNA-loaded chitosan-lipid nanocapsules decrease temozolomide resistance in glioblastoma: in vivo evaluation. Int J Pharm. 2015;481:154-161.

10. Trapani A, Denora N, Iacobellis G, Sitterberg J, Bakowsky U, Kissel T. Methotrexate-loaded chitosan- and glycol chitosan-based nanoparticles: a promising strategy for the administration of the anticancer drug to brain tumors. AAPS PharmSciTech. 2011;12:1302-1311.

11. Lollo G, Vincent M, Ullio-Gamboa G, et al. Development of multifunctional lipid nanocapsules for the co-delivery of paclitaxel and CpG-ODN in the treatment of glioblastoma. Int J Pharm. 2015;495:972-980.

12. Xu Y, Asghar S, Yang L, et al. Lactoferrin-coated polysaccharide nanoparticles based on chitosan hydrochloride/hyaluronic acid/PEG for treating brain glioma. Carbohydr Polym. 2017;157:419-428.

13. Barrow M, Taylor A, Nieves DJ, et al. Tailoring the surface charge of dextran-based polymer coated SPIONs for modulated stem cell uptake and MRI contrast. Biomater Sci. 2015;3:608-616.

14. Pattani A, Patravale VB, Paniker L, Potdar PD. Immune effects and membrane interaction of chitosan nanoparticles. Mol Phar. 2009;6 2345-2352.

15. Petri-Fink A, Hofmann H. Superparamagnetic iron oxide nanoparticles (SPIONS): from synthesis to in vivo studies - a summary of the synthesis, characterization, in vitro, and in vivo investigations of spions with particular focus on surface and colloidal properties. IEEE Trans Nanobioscience. 2007;6:289-297.

16. Shevtsov MA, Nikolaev BP, Ryzhov VA, et al. Ionizing radiation improves glioma-specific targeting of superparamagnetic iron oxide nanoparticles conjugated with cmHsp70.1 monoclonal antibodies (SPION-cmHsp70.1). Nanoscale. 2015;7:20652-20664.

17. Shevtsov MA, Nikolaev BP, Yakovleva LY, et al. Superparamagnetic iron oxide nanoparticles conjugated with epidermal growth factor (SPION-EGF) for targeting brain tumors. Int J Nanomedicine. 2014;9: 273-287.
18. Shevtsov MA, Nikolaev BP, Yakovleva LY, et al. 70-kDa heat shock protein coated magnetic nanocarriers as a nanovaccine for induction of anti-tumor immune response in experimental glioma. J Control Release. 2015;220:329-340.

19. Massart R. Preparation of aqueous magnetic liquids in alkaline and acidic media. IEEE Trans Magn. 1981;17:1247-1248.

20. Ryzhov VA, Larionov II, Fomichev VN. On the spurious signal in the longitudinal nonlinear susceptibility of magnets at the second harmonic of excitation frequency. Tech Phys. 1996;41:620-626.

21. Gullity BD, Graham CD. Introduction to Magnetic Materials. New York: IEEE Press. Wiley; 2009.

22. Ryzhov VA, Pleshakov IV, Nechitailov AA, et al. Magnetic study of nanostructural composite material based on cobalt compounds and porous silicon. Appl Magn Resonan. 2014;45:339-352.

23. Lazuta AV, Ryzhov VA, Runov VV, Khavronin VP, Deriglazov VV. Temperature evolution of superparamagnetic clusters in single-crystal $\mathrm{La}_{0.85} \mathrm{Sr}_{0.15} \mathrm{CoO}_{3}$ characterized by nonlinear magnetic ac response and neutron depolarization. Phys Rev B. 2015;92:14404-14411.

24. Bloembergen N, Purcell EM, Pound RV. Relaxation effects in Nuclear Magnetic Resonance Absorption. Phys Rev. 1948;73:679.

25. Abraham A. Principles of Nuclear Magnetism. Oxford: Clarendon Press; 1961:599.

26. Kittel C. Introduction to Solid State Physics. 8th ed. Indianapolis, IN: John Wiley \& Sons, Inc; 2005:690.

27. Ryzhov VA, Lazuta AV, Molkanov PI, et al. Comparative study of heterogeneous magnetic state above TC in $\mathrm{La} 0.82 \mathrm{Sr} 0.18 \mathrm{CoO} 3$ cobaltite and La0.83Sr0.17MnO3 manganite. JMMM. 2012;324:3432-3436.

28. Lazuta AV, Larionov II, Ryzhov VA. Second-harmonic nonlinear response of a cubic ferromagnet in the critical paramagnetic neighborhood of TC. Sov Phys JETP. 1991;73:1086-1095.

29. Ryzhov VA, Lazuta AV, Luzyanin ID, et al. The nonlinear magnetic properties of the pseudocubic $\mathrm{Nd} 0.77 \mathrm{Ba} 0.23 \mathrm{MnO} 3$ single crystal in the critical paramagnetic region and phase separation. JETP. 2002;94: 581-592.

30. Glaser T, Han I, Wu L, Zeng X. Targeted nanotechnology in glioblastoma multiforme. Front Pharmacol. 2017;8:166.

31. Pinto MP, Arce M, Yameen B, Vilos C. Targeted brain delivery nanoparticles for malignant gliomas. Nanomedicine (Lond). 2017;12:59-72.

32. Shevtsov M, Multhoff G. Recent developments of magnetic nanoparticles for theranostics of brain tumor. Curr Drug Metab. 2016;17:737-744.

33. Liu H, Gao C. Preparation and properties of ionically cross-linked chitosan nanoparticles. Poly Adv Technol. 2009;20:613-619.

34. Khan SA, Patil KS, Yeole PG. Intranasal mucoadhesive buspirone formulation: in vitro characterization and nasal clearance studies. Pharmazie Die. 2008;63:348-351.

35. Papadimitriou S, Bikiaris D, Avgoustakis K, Karavas E, Georgarakis M. Chitosan nanoparticles loaded with dorzolamide and pramipexole. Carbohydr Polym. 2008;73:44-54.

36. Jo J, Aoki I, Tabata Y. Design of iron oxide nanoparticles with different sizes and surface charges for simple and efficient labeling of mesenchymal stem cells. J Control Release. 2010;142:465-473.

37. Watanabe $\mathrm{M}$, Yoneda $\mathrm{M}$, Morohashi A, et al. Effects of $\mathrm{Fe} 3 \mathrm{O} 4$ magnetic nanoparticles on A549 cells. Int J Mol Sci. 2013;14:15546-15560.

38. Liu G, Gao J, Ai H, Chen X. Applications and potential toxicity of magnetic iron oxide nanoparticles. Small. 2013;9:1533-1545.

39. Esposito LA, Melov S, Panov A, Cottrell BA, Wallace DC. Mitochondrial disease in mouse results in increased oxidative stress. Proc Natl Acad Sci U S A. 1999;96:4820-4825.

40. Langkammer C, Krebs N, Goessler W, et al. Quantitative MR imaging of brain iron: a postmortem validation study. Radiology. 2010;257(2): $455-462$.

41. Haacke EM, Liu S, Buch S, Zheng W, Wu D, Ye Y. Quantitative susceptibility mapping: current status and future directions. Magn Reson Imaging. 2015;33(1):1-25.

42. Blasiak B, Barnes S, Foniok T, et al. Comparison of T2 and T2*weighted MR molecular imaging of a mouse model of glioma. $B M C$ Med Imaging. 2013;13:20. 
43. Blasiak B, Landry J, Tyson R, et al. Molecular susceptibility weighted imaging of the glioma rim in a mouse model. J Neurosci Methods. 2014; 226:132-138.

44. Haacke EM, Xu Y, Cheng YC, Reichenbach JR. Susceptibility weighted imaging (SWI). Magn Reson Med. 2004;52(3):612-618.

45. Matsumura Y, Maeda H. A new concept for macromolecular therapeutics in cancer-chemotherapy - mechanism of tumoritropic accumulation of proteins and the antitumor agent smancs. Cancer Res. 1986;46: 6387-6392.

46. Perrault SD, Walkey C, Jennings T, Fischer HC, Chan WCW. Mediating tumor targeting efficiency of nanoparticles through design. Nano Lett. 2009;9:1909-1915.

47. Wang B, Lv L, Wang Z, et al. Improved anti-glioblastoma efficacy by IL-13R $\alpha 2$ mediated copolymer nanoparticles loaded with paclitaxel. Sci Rep. 2015;5:16589.

48. Abakumov MA, Nukolova NV, Sokolsky-Papkov M, et al. VEGFtargeted magnetic nanoparticles for MRI visualization of brain tumor. Nanomedicine. 2015;11:825-833.

49. Hadjipanayis CG, Machaidze R, Kaluzova M, et al. EGFRvIII antibody-conjugated iron oxide nanoparticles for magnetic resonance imaging-guided convection-enhanced delivery and targeted therapy of glioblastoma. Cancer Res. 2010;70:6303-6312.
50. Tomanek B, Iqbal U, Blasiak B, et al. Evaluation of brain tumor vessels specific contrast agents for glioblastoma imaging. Neuro Oncol. 2012; 14(1):53-63.

51. Kratzer I, Wernig K, Panzenboeck U, et al. Apolipoprotein A-I coating of protamine-oligonucleotide nanoparticles increases particle uptake and transcytosis in an in vitro model of the blood-brain barrier. J Control Release. 2007;117(3):301-311.

52. Zensi A, Begley D, Pontikis C, et al. Albumin nanoparticles targeted with Apo E enter the CNS by transcytosis and are delivered to neurons. J Control Release. 2009;137:78-86.

53. Madhankumar AB, Slagle-Webb B, Mintz A, Sheehan JM, Connor JR. Interleukin-13 receptor-targeted nanovesicles are a potential therapy for glioblastoma multiforme. Mol Cancer Ther. 2006;5:3162-3169.

54. Liu L, Guo K, Lu J, et al. Biologically active core/shell nanoparticles self-assembled from cholesterol-terminated PEG-TAT for drug delivery across the blood-brain barrier. Biomaterials. 2008;29:1509-1517.
International Journal of Nanomedicine

\section{Publish your work in this journal}

The International Journal of Nanomedicine is an international, peerreviewed journal focusing on the application of nanotechnology in diagnostics, therapeutics, and drug delivery systems throughout the biomedical field. This journal is indexed on PubMed Central, MedLine, CAS, SciSearch ${ }^{\circledR}$, Current Contents ${ }^{\circledR} /$ Clinical Medicine,

\section{Dovepress}

Journal Citation Reports/Science Edition, EMBase, Scopus and the Elsevier Bibliographic databases. The manuscript management system is completely online and includes a very quick and fair peer-review system, which is all easy to use. Visit http://www.dovepress.com/ testimonials.php to read real quotes from published authors. 